\title{
EXTENSION OF AUSMPW+ SCHEME FOR TWO-FLUID MODEL
}

\author{
JIN SEOK PARK ${ }^{1}$ AND CHONGAM KIM ${ }^{1,2 \dagger}$ \\ ${ }^{1}$ Department of Aerospace Engineering, Seoul National University, South Korea \\ E-mail address: seok82@snu.ac.kr \\ ${ }^{2}$ Institute of Advanced Aerospace Technology, Department of Aerospace Engineering, \\ SEOUl National University, SOUth Korea \\ E-mail address: chongamesnu.ac.kr
}

\begin{abstract}
The present paper deals with the extension of AUSMPW+ scheme into two-fluid model for multiphase flow. AUSMPW+ scheme is the improvement of a single-phase AUSM+ scheme by designing pressure-based weighting functions to prevent oscillations near a wall and shock instability after a strong shock. Recently, Kitamura and Liou assessed a family of AUSM-type schemes with two-fluid model governing equations [K. Kitamura and M.-S. Liou, Comparative study of AUSM-Family schemes in compressible multi-phase flow simulations, ICCFD7-3702 (2012)]. It was observed that the direct application of the single-phase AUSMPW+ did not provide satisfactory results for most of numerical test cases, which motivates the current study. It turns out that, by designing pressure-based weighting functions, which play a key role in controlling numerical diffusion for two-fluid model, problems reported in can be overcome. Various numerical experiments validate the proposed modification of AUSMPW+ scheme is accurate and robust to solve multiphase flow within the framework of two-fluid model.
\end{abstract}

\section{INTRODUCTION}

Recently, analyzing multi-phase flow becomes popular due to various applications, such as cavitating flow in hydraulic machine, high-speed underwater projectiles and liquid-gas interactions. There have been many numerical studies, such as interface tracking methods and interface capturing methods. In order to handle complex geometry and phase transition, two interface capturing methods are available: homogeneous equilibrium model (HEM) and two-fluid model. While HEM is more efficient and provides reasonable solution for many applications, two-fluid model is more favorable in describing inter-phasic flow physics.

There are, however, many numerical difficulties to compute two-fluid model. Most notably, while governing equation for each phase is hyperbolic, combining them into two-fluid model becomes non-hyperbolic. In addition, wave speed for each phase is radically different, yielding a very stiff mathematical from. Finally, the non-conservative term may give erroneous results

Received by the editors June 8 2013; Accepted July 292013.

2000 Mathematics Subject Classification. 76M12, 76T10.

Key words and phrases. Multiphase flow, Two-fluid Model, AUSMPW+ scheme.

${ }^{\dagger}$ Corresponding author. 
when the conservative scheme, which is typically used in single phase fluid, is applied. Thus, proper numerical algorithm for two-fluid model is necessary.

Various approaches are developed to overcome the above-mentioned difficulties. Recently, Liou et al. proposed a new approach based on stratified flow model [1,2] and AUSM+-up scheme [3]. By successfully extending single-phase AUSM-type flux scheme into two-fluid model, this method does not requires the exact Riemann solver.

Many AUSM-type flux schemes can be easily adopted and recently their performance were compared. Although AUSMPW+ scheme is one of the most shock-stable and accurate flux in compressible flows $[4,5]$, direct application to two-fluid model fails to compute most of test cases [6]. This is mainly attributed that the pressure-based weighting functions, the key part of AUSMPW+ scheme, is improperly working. These functions, based on the compressible flow physics, are originally devised to eliminate the overshoots behind strong shock and/or oscillations near a wall, which is a typical symptom appearing in most AUSM-type schemes. It is also studied that the modifications of the pressure-based weighting functions functions are necessary to extend AUSMPW+ scheme to HEM-based multiphase flows [7] and magnetohydrodynamics (MHD) [8].

The present paper deals with the extension of AUSMPW+ scheme for two-fluid model. At first, two-fluid model and its numerical treatment are briefly introduced as a baseline approach. Examining the shock-stability mechanism of AUSMPW+ scheme, the proper extension for two-fluid model is proposed. Extensive numerical experiments are conducted and conclusions are given.

\section{Baseline Approach: Two-Fluid Model}

2.1. Governing Equations. In the two-fluid model, the governing equations for each phase are directly solved.

$$
\begin{gathered}
\frac{\partial \mathbf{Q}_{k}}{\partial t}+\nabla \cdot \mathbf{F}_{k}=\mathbf{P}_{k}^{i n t}+\mathbf{S}_{k}, k=g, l \\
\mathbf{Q}_{k}=\left(\begin{array}{c}
\alpha \rho \\
\alpha \rho \mathbf{V} \\
\alpha \rho E
\end{array}\right)_{k}, \mathbf{F}_{k}=\left(\begin{array}{c}
\alpha \rho \mathbf{V} \\
\alpha \rho \mathbf{V} \cdot \mathbf{V}+\alpha p \\
\alpha \rho \mathbf{V} H
\end{array}\right)_{k}, \mathbf{P}_{k}^{i n t}=p^{i n t}\left(\begin{array}{c}
0 \\
\nabla \alpha \\
\frac{\partial \alpha}{\partial t}
\end{array}\right)_{k},
\end{gathered}
$$

where $g$ and $l$ indicate the gas and liquid phase, respectively. $\mathbf{Q}_{k}, \mathbf{F}_{k}$ and $\mathbf{S}_{k}$ are the conservative vector, inviscid flux vector and volumetric source vector (due to body foces, heat addition, phase change, etc.), respectively. $\alpha_{k}$ is the volume fraction with $\alpha_{l}+\alpha_{g}=1$. To describe the interaction between gas and liquid phase, the inter-facial pressure vector $\mathbf{P}_{k}^{i n t}$ is introduced.

It is assumed that the bulk pressure and inter-facial pressure of each phase are the same.

$$
\begin{array}{r}
p_{l}=p_{g}=p, \\
p_{l}^{i n t}=p_{g}^{i n t}=p^{i n t} .
\end{array}
$$

In addition, the inter-facial pressure is expressed with the bulk pressure and finite jump term [9].

$$
p^{i n t}=p-\Delta p^{*} .
$$


For liquid and gas system, it can be usually formulated as follows.

$$
\Delta p^{*}=\sigma \frac{\alpha_{g} \alpha_{l} \rho_{g} \rho_{l}}{\alpha_{g} \rho_{l}+\alpha_{l} \rho_{g}}\left|\mathbf{V}_{l}-\mathbf{V}_{g}\right|^{2} .
$$

Assuming that each phase is dispersed and continuous, it can be simplified as follows.

$$
\Delta p^{*}=C_{p}^{*} \alpha_{l} \alpha_{g}\left|\mathbf{V}_{l}-\mathbf{V}_{g}\right|^{2}
$$

While each phase are governed by hyperbolic conservation laws, the two-fluid model (Eq. (2.1)) may lose hyperbolicity. In order to keep the system hyperbolic, coefficient of the interfacial pressure $\left(\sigma, C_{p}^{*}\right)$ becomes large [2]. In the present computations, the inter-facial pressure term is modeled by Eq. (2.6) with $C^{*}=2.0$.

To close the system, the equation of state (EOS) for each phase is required. The ideal gas EOS and stiffened EOS [11] are adopted for gas and liquid phase, respectively.

$$
p_{k}=\rho_{k} \frac{\gamma_{k}-1}{\gamma_{k}} C_{p k} T_{k}-p_{k \infty}, e_{k}=\frac{C_{p k}}{\gamma_{k}} T_{k}+\frac{p_{k \infty}}{\rho_{k}},
$$

where $\gamma_{g}=1.4, C_{p g}=1004.5 \mathrm{~J} /(\mathrm{kgK}), p_{g \infty}=0 P a$ and $\gamma_{l}=2.8, C_{p l}=4186 \mathrm{~J} /(\mathrm{kgK})$, $p_{l \infty}=8.5 \times 10^{8} \mathrm{~Pa}$.

2.2. Stratified Flow Model and Spatial Discretization. In order to discretize the governing equation (Eq. (2.1)), we adopt the stratified flow model [10,1]. This model divides each phase region according to characteristic property, such as volume fraction. This concept is suitable to the finite volume discretization, and we can construct two control volumes for liquid and gas within a cell. It is assumed that the volume fraction $\alpha_{k}$ is piecewise continuous within the cell and allowed a jump at a cell interface. For each phase, the conservation laws are applied as in the single phase fluids, and the interactions at the sub-cell interface between gas and liquid phases are additionally considered. The present computations ignore phase transition and viscous effects, and thus only inter-facial pressure affects the momentum and energy conservation as follows.

$$
\frac{\Delta x}{\Delta t} \delta^{t}\left(\begin{array}{c}
\alpha_{k} \rho_{k} \\
\alpha_{k} \rho_{k} u_{k} \\
\alpha_{k} \rho_{k} E_{k}
\end{array}\right)_{j}+\delta_{j}^{x}\left(\begin{array}{c}
\alpha_{k} \rho_{k} u_{k} \\
\alpha_{k} \rho_{k} u_{k}^{2}+\alpha_{k} p \\
\alpha_{k} \rho_{k} u_{k} H_{k}
\end{array}\right)-p_{j}^{i n t}\left(\begin{array}{c}
0 \\
\delta_{j}^{x}\left(\alpha_{k, j}\right) \\
\frac{\Delta x}{\Delta t} \delta^{t}\left(\alpha_{k, j}\right)
\end{array}\right)=0 .
$$

where $\delta^{t}(\cdot)=(\cdot)^{n+1}-(\cdot)^{n}$ and $\delta_{j}^{x}(\cdot)=(\cdot)_{j+1 / 2}-(\cdot)_{j-1 / 2}$.

The inter-facial pressure affects the momentum conservation if the properties (including $\alpha_{k}$ ) are discretized by at least a second-order accurate method. Here, a second-order accurate MUSCL interpolation is used. At the cell interface, monotone and accurate numerical flux scheme is required, which is going to be discussed in the next section.

2.3. Time Marching and Solution Update. In order to update solution variables, third-order accurate TVD Runge-Kutta method is used. The time-derivative term appearing in the interfacial pressure vector is included in time marching, and thus the energy equation is computed 
as follows.

$$
\begin{array}{r}
\hat{\mathbf{Q}}_{3, k}=\mathbf{Q}_{3, k}+\alpha_{k} p^{i n t} \\
\frac{\partial \hat{\mathbf{Q}}_{3, k}}{\partial t}+\sum_{\text {face }}\left(\mathbf{F}_{3, k} \cdot \mathbf{n}\right) l=0
\end{array}
$$

where $\mathbf{n}$ and $l$ are the normal vector and length of the face, respectively. After time marching, the decoding process is required to update $p^{n+1}$ and $\alpha_{k}^{n+1}$.

$$
\begin{array}{r}
\left(p+\hat{a}_{k}\right) \alpha_{k}-\hat{A}_{k}=0, \\
\hat{a}_{k}=\gamma_{k} p_{k, \infty}+\left(\gamma_{k}-1\right) p^{i n t}, \\
\hat{A}_{k}=\left(\gamma_{k}-1\right)\left(\hat{\mathbf{Q}}_{3, k}-\frac{\mathbf{Q}_{2, k}^{2}}{2 \mathbf{Q}_{1, k}}\right) .
\end{array}
$$

Equation (2.11) are then solved by the Newton iteration method [6].

\section{AUSMPW + SCHEME FOR TWO-FLUID MODEL}

3.1. Baseline Scheme: AUSMPW+. The AUSMPW+ scheme for a single gas phase with $k=g$ can be formulated as follows.

$$
\mathbf{F}_{k, 1 / 2, L / R}=\bar{M}_{k, L}^{+} c_{k, 1 / 2} \boldsymbol{\Phi}_{k, L}+\bar{M}_{k, R}^{-} c_{k, 1 / 2} \boldsymbol{\Phi}_{k, R}+\alpha_{k, 1 / 2, L / R}\left(P_{k, L}^{+} \mathbf{P}_{L}+P_{k, R}^{+} \mathbf{P}_{R}\right),
$$

where $\boldsymbol{\Phi}=(\alpha \rho, \alpha \rho \mathbf{V}, \alpha \rho H)^{T}$ and $P=(0, p \mathbf{n}, 0)^{T}$.

For $m_{1 / 2}=m_{1 / 2}=M_{k, L}^{+}+M_{k, R}^{-} \geq 0$,

$$
\begin{aligned}
& \bar{M}_{k, L}^{+}=M_{k, L}^{+}+M_{k, R}^{-} \cdot\left[\left(1-w_{k}\right) \cdot\left(1+f_{k, R}\right)-F_{k, L}\right], \\
& \bar{M}_{k, R}^{-}=M_{k, R}^{-} \cdot w_{k} \cdot\left(1+f_{k, R}\right) .
\end{aligned}
$$

For $m_{1 / 2} \leq 0$,

$$
\begin{aligned}
& \bar{M}_{k, L}^{+}=M_{k, L}^{+} \cdot w_{k} \cdot\left(1+f_{k, L}\right), \\
& \bar{M}_{k, R}^{-}=M_{k, R}^{+}+M_{k, L}^{+} \cdot\left[\left(1-w_{k}\right) \cdot\left(1+f_{k, L}\right)-F_{k, R}\right] .
\end{aligned}
$$

We use the AUSM-type interpolating functions for the $\operatorname{Mach} \operatorname{number}(M)$ and pressure $(P)$ [4], as follows.

$$
\begin{aligned}
& M_{k}^{ \pm}=\left(\begin{array}{cl} 
\pm \frac{1}{4}\left(M_{k} \pm 1\right)^{2}, & \left|M_{k}\right| \leq 1 \\
\frac{1}{2}\left(M_{k} \pm\left|M_{k}\right|\right), & \left|M_{k}\right|>1
\end{array}\right. \\
& \left.P^{ \pm}\right|_{k, L / R}=\left(\begin{array}{ll} 
\pm \frac{1}{4}\left(M_{k} \pm 1\right)^{2}\left(2 \mp M_{k}\right) \pm \alpha M_{k}\left(M_{k}^{2}-1\right)^{2}, & \left|M_{k}\right| \leq 1 \\
\frac{1}{2}\left(1 \pm \operatorname{sign}\left(M_{k}\right)\right), & \left|M_{k}\right|>1
\end{array}\right.
\end{aligned}
$$

The speed of sound at a cell-interface is given by

$$
\begin{gathered}
c_{k, 1 / 2}=\frac{c_{k, s}^{2}}{\max \left(\left|U_{k, L / R}\right|, c_{k, s}\right)}, c_{k, s}=\sqrt{\frac{2(\gamma-1)}{(\gamma+1)} H_{k, n},} \\
H_{k, n}=0.5 \times\left(H_{k, L}-0.5 V_{L}^{2}+H_{k, R}-0.5 V_{R}^{2}\right),
\end{gathered}
$$


where $U$ is the contravariant velocity and $V$ is the tangential velocity.

In order to treat oscillations near a wall and across a strong shock, two pressure-based functions, $f$ and $w$, are introduced as follows.

$$
\begin{aligned}
& f_{k, L / R}=\left\{\begin{array}{ll}
\left(\frac{p_{L, R}}{p_{k, s}}-1\right) w_{2}, & p_{k, s} \neq 0 \\
0, & \text { elsewhere }
\end{array}, w_{2}=\min \left(1, \frac{\min \left(p_{1, L}, p_{1, R}, p_{2, L}, p_{2, R}\right)}{\min \left(p_{L}, p_{R}\right)}\right)^{2},\right. \\
& w\left(p_{L}, p_{R}\right)=1-\min \left(\frac{p_{L}}{p_{R}}, \frac{p_{R}}{p_{L}}\right)^{3},
\end{aligned}
$$

where $p_{k, s}=P_{k, L}^{+} p_{L}+P_{k, R}^{-} p_{R}$.

3.2. Analysis and Modification of Pressure-based Weighting Functions. Equations (3.8) and (3.9) basically control the amount of numerical diffusion caused by the pressure difference and difference of advection term. Kitamura and Liou extended a single gas phase AUSMPW+ into two-fluid model without any modification [6], and it was observed that the two functions are unstable for two-fluid model, leading to divergence of computations in most test cases. According to authors' experience on the extension of AUSMPW+ for HEM multiphase flow and MHD, it is essential to modify these terms based on its diffusion mechanism and its design principle. We start it by examining each numerical diffusion term.

For $0 \leq m_{1 / 2} \leq 1$, the diffusion term of AUSMPW+ scheme can be written as follows.

$$
\begin{array}{r}
D_{A U S M P W+}=U_{k, R} \Delta \Phi_{k}+\frac{U_{k, L}+U_{k, R}}{2 c_{k, 1 / 2}} \Phi_{k, L} \Delta U_{k}-\left(P_{k, L}^{+} p_{L}+P_{k, R}^{-} p_{R}\right) \\
+\frac{c_{k, 1 / 2}}{2}\left(M_{k, R}-1\right)^{2} w\left(1+f_{k, R}\right) \Delta \Phi_{k}+\frac{c_{k, 1 / 2}}{2}\left(M_{k, R}-1\right)^{2} \Phi_{k, L} \Delta f_{k} .
\end{array}
$$

Comparing Eq. (3.10) to the diffusion term of AUSM+-up [3] scheme, we can observe following characteristics.

$$
\begin{gathered}
D_{A U S M^{+-u p}}=U_{k, R} \Delta \Phi_{k}+\frac{U_{k, L}+U_{k, R}}{2 c_{k, 1 / 2}} \Phi_{k, L} \Delta U_{k}-M_{p k} c_{k, 1 / 2} \Phi_{k, L}-\left(P_{k, L}^{+} p_{L}+P_{k, R}^{-} p_{R}-p_{u k}\right) . \\
M_{p k}=-\frac{K_{p}}{f_{a}} \max \left(1-\bar{M}_{k}^{2}, 0\right) \frac{\Delta p}{\overline{\rho_{k}} c_{k, 1 / 2}^{2}}, p_{u k}=-K_{u} P_{k, L}^{+}\left|P_{k, R}^{-}\right| \bar{\rho}_{k} f_{a} c_{k, 1 / 2} \Delta U_{k},
\end{gathered}
$$

where $K_{p}$ and $K_{u}$ are coefficients. $f_{a}$ is a scaling function for all-speed extension but it is needless for two-fluid model (i.e. $f_{a}=1$ ).

i) Both scheme (AUSMPW+ and AUSM+-up) have the numerical diffusion due to pressure difference, and they are similar to each other as follows.

$$
\begin{aligned}
D_{A U S M P W+}^{(p)} & =\frac{c_{k, 1 / 2}}{2}\left(M_{k, R}-1\right)^{2} \Phi_{k, L} \Delta f_{k}=\frac{c_{k, 1 / 2}}{2}\left(M_{k, R}-1\right)^{2} w_{2} \Phi_{k, L} \frac{\Delta p}{p_{k, s}} \\
D_{A U S M+-u p}^{(p)} & =-M_{p k} c_{k, 1 / 2} \Phi_{k, L}=\frac{K_{p}}{f_{a}} c_{k, 1 / 2}\left(1-\bar{M}_{k}^{2}\right) \Phi_{k, L} \frac{\Delta p}{\bar{\rho}_{k} c_{k, 1 / 2}^{2}}
\end{aligned}
$$


ii) While AUSMPW+ scheme has additional diffusion mechanism due to jump in advection variable $\left(\Delta \Phi_{k}\right)$, AUSM+-up scheme adjusts the pressure flux by the difference in interface normal velocity.

The role of $f$ of AUSMPW+ is a kind of bridge between AUSM+ and AUSMD. While it consists of pressure ratio, it actually reflects the density difference via the definition of speed of sound (see Eqs (14-17) in Ref [4]).

$$
\begin{aligned}
(\rho u)_{k, 1 / 2, A U S M P W+} & =M_{k, L}^{+} c_{k, 1 / 2} \rho_{k, L}+M_{k, R}^{-} c_{k, 1 / 2} \rho_{k, L}\left(\frac{\rho_{k, R}}{\rho_{k, L}}\right) \\
& \approx M_{k \cdot L}^{+} c_{k, 1 / 2} \rho_{k, L}+M_{k \cdot R}^{-} c_{k, 1 / 2} \rho_{k, L}\left(\frac{p_{R}}{p_{L}}\right) .
\end{aligned}
$$

To be consistent with the stiffened EOS [10], the pressure ratio of $f$ (Eq. (3.8)) has to be written in terms of $\bar{p}=p+p_{\infty}$.

$$
f_{k, L / R}= \begin{cases}\left(\frac{\bar{p}_{L / R}}{\bar{p}_{s}}-1\right) w_{2} & p_{s} \neq 0 \\ 0 & \text { else }\end{cases}
$$

Furthermore, the term $w_{2}$ in Eq. (3.8), which is designed to detect stagnation region of boundary layer or shock in multi-dimensional situation, also controls the magnitude of numerical diffusion by pressure jump (see Eq. (3.13)). The problem is that, while $w_{2}$ successfully controls the diffusion by pressure jump for gas phase, it does not provide adequate diffusion for liquid phase or transition of phase. From this perspective, $w_{2}$ is also computed using $\bar{p}$.

$$
w_{2}=\min \left(1, \frac{\min \left(\bar{p}_{1, L}, \bar{p}_{1, R}, \bar{p}_{2, L}, \bar{p}_{2, R}\right)}{\min \left(\bar{p}_{L}, \bar{p}_{R}\right)}\right)^{2}
$$

On the other hand, the role of $w$ is to detect a large pressure jump (especially around shock) and introduces additional diffusion to remove spurious oscillation (see the fourth term in Eq. (3.10)). If this term is also replaced by $\bar{p}, w$ cannot detect pressure jump in liquid phase and cannot suppress oscillations by pressure jump. Thus, $w$ should be kept as the original form Eq. (3.9), and thus it is the same in both gas and liquid phase.

While AUSMPW+ for HEM model additionally introduces a term based on mixture density in phase transition (Eq. (46) in Ref. [7]) to control the fourth term in Eq. (3.10), AUSMPW+ for two-fluid model does not require such device. AUSM+-up and other AUSM-type scheme (for example, SLAU [12]) control the pressure flux by velocity difference (Eq.(3.12)). Though not essential, $p_{u k}$ could be a little bit helpful in suppressing a slight kink after shock in liquid phase. Thus, we add $p_{u k}$ in the pressure flux of AUSMPW+.

In summary, by examining the behavior of numerical diffusion, AUSMPW+ for two-fluid model can be obtained by replacing $p$ of Eq. (3.8) by $\bar{p}$. The usage of $p_{u k}$ of Eq. (3.12) is optional. 


\section{NUMERICAL EXPERIMENTS}

We use a two-dimensional structured finite volume solver. To account for the inter-facial pressure, a second-order MUSCL interpolation with minmod limiter is applied to the primitive variables $Q_{p}=\left(\alpha_{g}, u_{g}, u_{l}, v_{g}, v_{l}, P, T_{g}, T_{l}\right)$. With the developed AUSMPW+ scheme, we compute four test cases. Without the proposed modification, it is reported that AUSMPW+ fails to solve them [6]. All computed results are compared with AUSM+ and AUSM+-up(1,1). Hereafter, AUSM+-up indicates AUSM+-up(1,1).

4.1. Moving Phase Discontinuity. The first test case is to examine the capability to resolve phase discontinuity maintaining the pressure. Computational domain is $[0,10] \times[0,1]$ with $\Delta x=\Delta y=0.05$. Initially, air and water is separated at $x=5$.

$$
\begin{aligned}
\left(p, \alpha_{g}, u_{k}, v, k, T_{k}\right)_{L} & =\left(10^{5} \mathrm{~Pa}, 1-\epsilon, 100 \mathrm{~m} / \mathrm{s}, 0 \mathrm{~m} / \mathrm{s}, 300 \mathrm{~K}\right) \text { for } x \leq 5 \mathrm{~m}, \\
\left(p, \alpha_{g}, u_{k}, v, k, T_{k}\right)_{R} & =\left(10^{5} \mathrm{~Pa}, \epsilon, 100 \mathrm{~m} / \mathrm{s}, 0 \mathrm{~m} / \mathrm{s}, 300 \mathrm{~K}\right) \text { else },
\end{aligned}
$$

where $k=l, g$ and $\epsilon=1.0 \times 10^{-7}$.

Figure 1 shows the distributions of pressure and void fraction along the centerline. All flux schemes can compute the moving phase transition successfully.

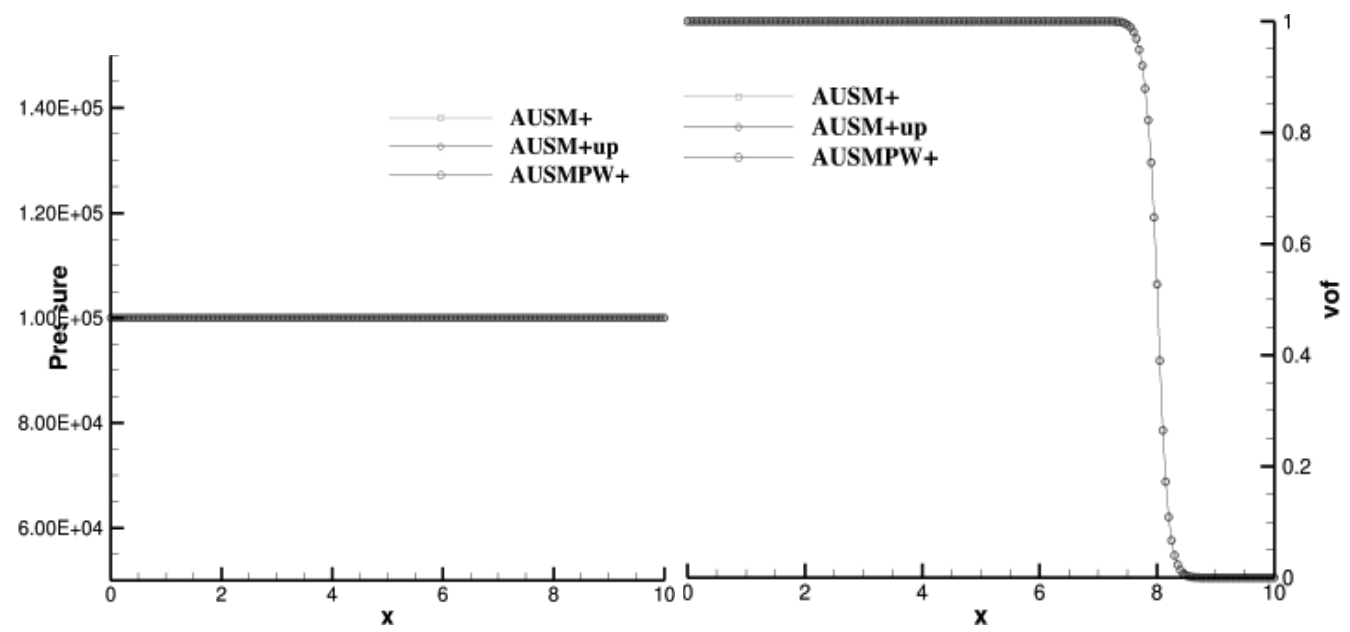

(a) Pressure

(b) Volume of Fraction

FIGURE 1. Distribution of pressure and volume of fraction along the centerline at $t=0.03 \mathrm{~s}$.

4.2. Shock Tube Problem. In order to examine robustness across underwater shock waves, two kinds of shock tube problems are considered. We conduct the propagation of shock wave from air to water and vice versa. Computational domain is $[0,10] \times[0,1]$ and $\Delta x=\Delta y=0.02$. Two initiail conditions are imposed as follows. 
Air-to-Water Shock Tube

$$
\begin{aligned}
\left(p, \alpha_{g}, u_{k}, v_{k}, T_{k}\right)_{L} & =\left(10^{9} p a, 1-\epsilon, 100 \mathrm{~m} / \mathrm{s}, 0 \mathrm{~m} / \mathrm{s}, 308.15 \mathrm{~K}\right) \text { for } x \leq 5 \mathrm{~m}, \\
\left(p, \alpha_{g}, u_{k}, v_{k}, T_{k}\right)_{R} & =\left(10^{5} \mathrm{pa}, \epsilon, 100 \mathrm{~m} / \mathrm{s}, 0 \mathrm{~m} / \mathrm{s}, 308.15 \mathrm{~K}\right) \text { else }
\end{aligned}
$$

Water-to-Air Shock Tube

$$
\begin{aligned}
& \left(p, \alpha_{g}, u_{k}, v_{k}, T_{k}\right)_{L}=\left(10^{7} p a, \epsilon, 100 \mathrm{~m} / \mathrm{s}, 0 \mathrm{~m} / \mathrm{s}, 308.15 \mathrm{~K}\right) \text { for } x \leq 5 \mathrm{~m}, \\
& \left(p, \alpha_{g}, u_{k}, v_{k}, T_{k}\right)_{R}=\left(5 \times 10^{6} p a, 1-\epsilon, 100 \mathrm{~m} / \mathrm{s}, 0 \mathrm{~m} / \mathrm{s}, 308.15 \mathrm{~K}\right) \text { else, }
\end{aligned}
$$

where $k=l, g$ and $\epsilon=1.0 \times 10^{-7}$.

Figures 2 and 3 show the pressure distribution of air-to-water shock tube and water-toair shock tube, respectively. The dotted line represents the gas volume fraction. AUSM+ suffers spurious oscillations around shock region in water, especially for water-to-air shock tube. AUSMPW+ with the proposed modification and AUSM+-up $(1,1)$ can successfully resolve these wave structures.

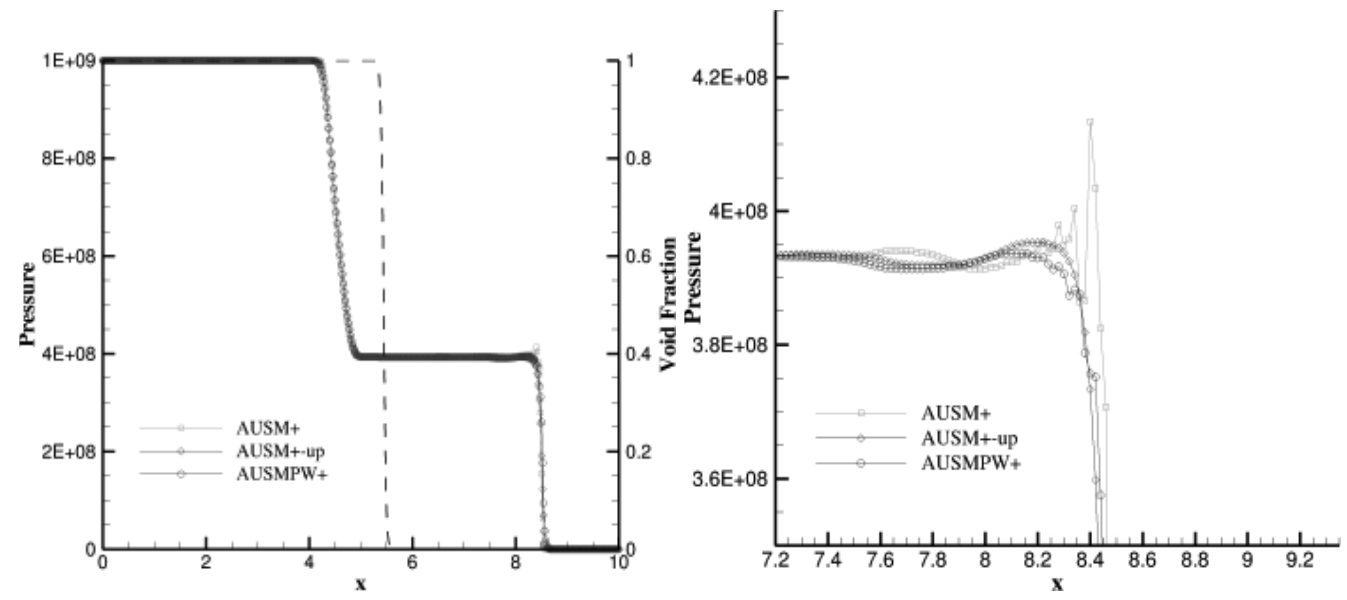

FIGURE 2. Distribution of pressure along the centerline at $t=0.002 s$. (Right: Close-up view around shock region)

4.3. One-dimensional Cavitation Problem. This is a simplified cavitation problem, proposed by Saurel and Abgrall [13]. After two opposite rarefaction waves are propagating in tube, velocity reaches zero at the center of domain. Thus, liquid flow containing $1 \%$ gas creates a cavitation zone at the center. Computational domain is $[0,10] \times[0,1]$ with $\Delta x=\Delta y=0.05$. The initial condition is

$$
\begin{aligned}
\left(p, \alpha_{g}, u_{k}, v_{k}, T_{k}\right)_{L} & =\left(10^{5} p a, \epsilon,-100 \mathrm{~m} / \mathrm{s}, 0 \mathrm{~m} / \mathrm{s}, 300 \mathrm{~K}\right) \text { for } x \leq 5 \mathrm{~m} \\
\left(p, \alpha_{g}, u_{k}, v_{k}, T_{k}\right)_{R} & =\left(10^{5} \mathrm{pa}, \epsilon, 100 \mathrm{~m} / \mathrm{s}, 0 \mathrm{~m} / \mathrm{s}, 300 \mathrm{~K}\right) \text { else }
\end{aligned}
$$

where $k=l, g$ and $\epsilon=1.0 \times 10^{-2}$. 


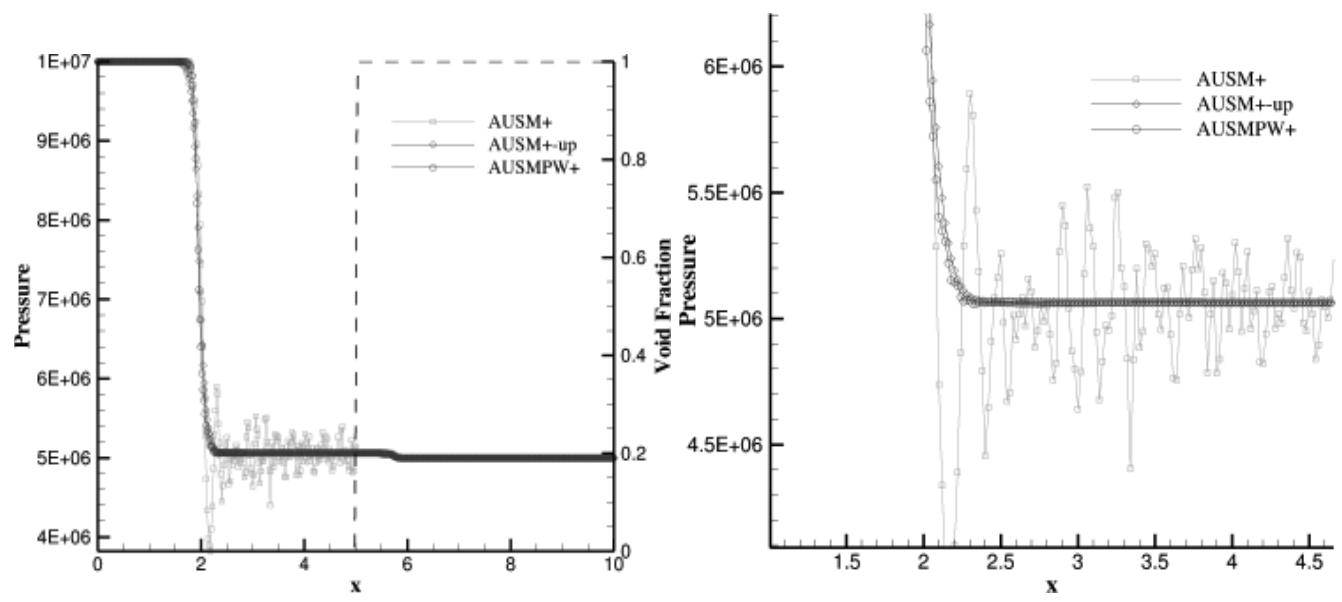

FIGURE 3. Distribution of pressure along the centerline at $t=0.002 s$. (Right: Close-up view around shock region)

Figure 4 shows the pressure and void fraction of AUSM+-up(1,1) and AUSMPW+ scheme. Both solutions are almost identical.

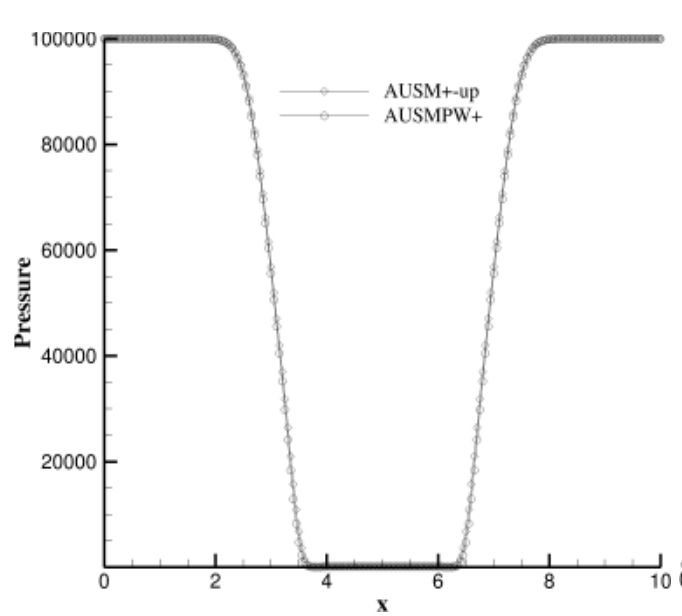

(a) Pressure

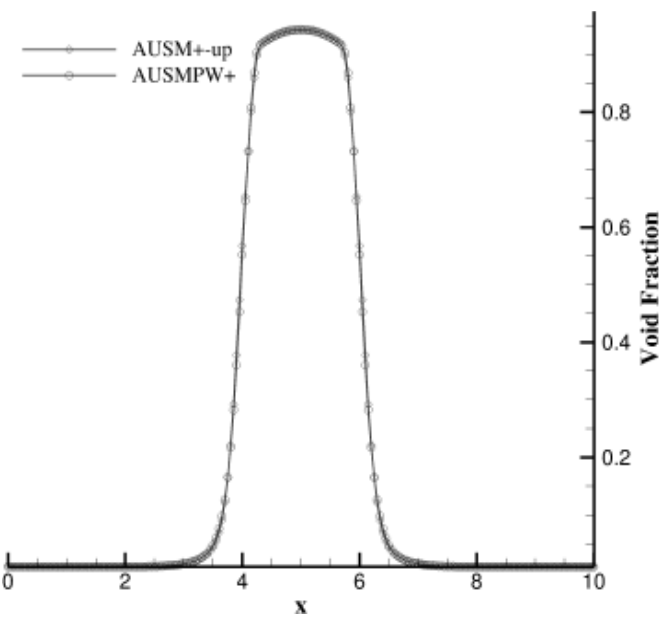

(b) Void Fraction

FIGURE 4. Distribution of pressure and void fraction along the centerline at $t=10 \mu s)$

4.4. Shock/Water-Column Interaction. The last test is more challenging and realistic problem. When shock wave impinges on the water column, shock wave transmits into water and travels faster. Shock wave in water also reflects at the rear interface. Considering the flow symmetry, only the upper half region is simulated. Computational domain is $[-15 \mathrm{~mm}, 20 \mathrm{~mm}] \times$ 
$[0 \mathrm{~mm}, 15 \mathrm{~mm}]$, and discretized by $400 \times 200$ cells. The initial condition is provided as follows. $\left(p, \alpha_{g}, u_{k}, v_{k}, T_{k}\right)=\left(2.35438 \times 10^{5} \mathrm{pa}, \epsilon, 225.86 \mathrm{~m} / \mathrm{s}, 0 \mathrm{~m} / \mathrm{s}, 381.85 \mathrm{~K}\right)$ for $x \leq-4 \mathrm{~mm}$, $\left(p, \alpha_{g}, u_{k}, v_{k}, T_{k}\right)=\left(10^{5} p a, 1-\epsilon, 0 \mathrm{~m} / \mathrm{s}, 0 \mathrm{~m} / \mathrm{s}, 293.15 K\right)$ for $x \geq-4 \mathrm{~mm}$,

$$
\sqrt{\left(x^{2}+y^{2}\right)} \leq 3.2 m m
$$

$\left(p, \alpha_{g}, u_{k}, v_{k}, T_{k}\right)=\left(10^{5} p a, \epsilon, 0 \mathrm{~m} / \mathrm{s}, 0 \mathrm{~m} / \mathrm{s}, 293.15 \mathrm{~K}\right)$ else,

where $k=l, g$ and $\epsilon=1 \times 10^{-5}$.

Figure 5 shows the pressure contours at $t=10 \mu \mathrm{s}$. The solid line is the boundary of water column. AUSMPW+ successfully captures the transmission and diffraction of the impinging shock wave and the transmitted shock reflected from the rear water column. These results are quite similar to other AUSM-type results $[2,6]$.

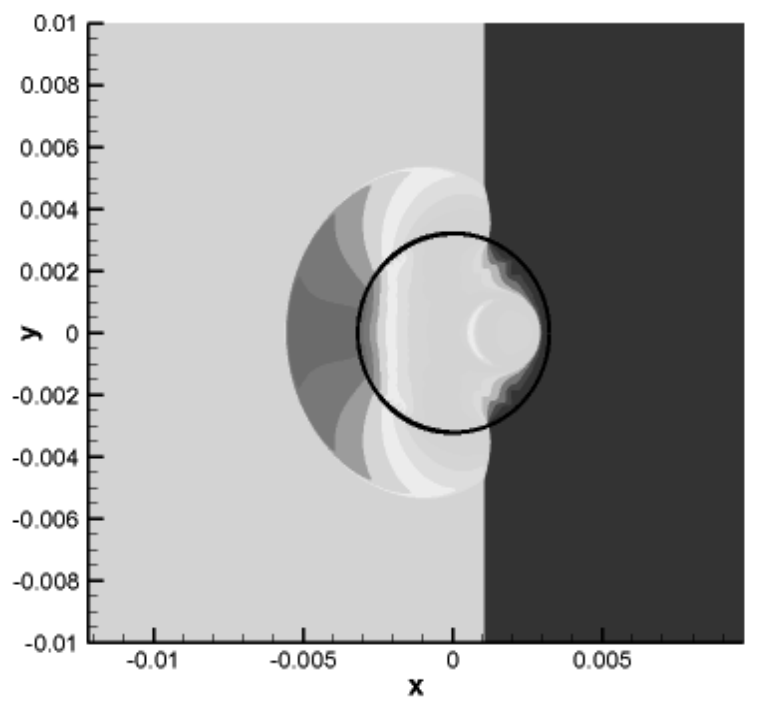

FIGURE 5. Pressure contours at $t=10 \mu \mathrm{s}$

\section{CONCLUSiON}

In this paper, we successfully extend AUSMPW+ scheme to two-fluid model. By examining the behavior of numerical diffusion, AUSMPW+ scheme for two-fluid model can be obtained by simply changing the pressure-based weighting function $f$ only. All computed results for critical test cases confirms that AUSMPW+ provides robust and accurate solutions of compressible two-fluid multiphase flow with shock waves. 


\section{ACKNOWLEDGMENTS}

Authors appreciate the financial supports provided by NSL (National Space Lab.) program through the National Research Foundation of Korea funded by the Ministry of Education, Science and Technology (Grant 2012-0009099) and by the EDISON program through the National Research Foundation of Korea(NRF) funded by the Ministry of Education, Science and Technology (Grant 2012-0006661).

\section{REFERENCES}

[1] M.-S. Liou and C.-H. Chang, A robust and accurate approach to computing compressible multiphase flow: Stratified flow model and AUSM+-up scheme, Journal of Computational Physics, 225, (2007), 840-873.

[2] M.-S. Liou, C.-H. Chang, C.-L. Nguyen and T. G. Theofanous, How to Solve Compressible Multifluid Equations: A Simple, Robust, and Accurate Method, AIAA Journal, 46, (2008), 2345-2356.

[3] M.-S. Liou, A Squel to AUSM, Part II: AUSM+up for all speeds, Journal of Computational Physics, 214, (2006), 137-170

[4] K. H. Kim, C. Kim and O. H. Rho, Methods for the accurate computations of hypersonic flows: I. AUSMPW+Scheme, Journal of Computational Physics, 174, (2001), 38-80.

[5] K. H. Kim and C. Kim, Accurate, efficient and monotonic numerical methods for multi-dimensional compressible flows: Part I: Spatial discretization, Journal of Computational Physics, 208, (2005), 527-569.

[6] K. Kitamura and M.-S. Liou, Comparative Study of AUSM-Family Schemes in Compressible Multiphase Flow Simulations, Proceedings of ICCFD7, Big Island, Hawaii 2012.

[7] S.-W. Ihm and C. Kim, Computations of homogeneous-equilibrium two-phase flows with accurate and efficient shock-stable schemes, AIAA Journal, 46, (2008), 3012-3037.

[8] S.-H. Han, J.-I Lee and K. H. Kim, Accurate and robust pressure weight advection upstream splitting method for magnetohydrodynamics equations, AIAA Journal, 47, (2009), 970-981.

[9] J. Stuhmiller, The Influence of Interfacial Pressure Forces on the Character of Two-Phase Flow Model Equations, International Journal of Multiphase Flow, 3, (1977), 551-560.

[10] H. B. Stewart, and B. Wendroff, Two-Phase Flow: Models and Methods, Journal of Computational Physics, 3, (1984), 363-409.

[11] F. Harlow, and A. Amsden, Fluid Dynamics, Los Alamos Scientific Lab., Rept. LA-4700, Los Alamos, NM, 1971.

[12] E. Shima and K. Kitamura, Parameter-Free Simple Low-Dissipation AUSM-Family Scheme for All Speeds, AIAA Journal 49, (2011), 1693-1709.

[13] R. Saurel and R. Abgrall, A Multiphase Godunov Method for Compressible Multifluid and Multiphase Flows, Journal of Computational Physics, 150, (1999), 425-467. 